\title{
Efficacy and Mechanism of Panax Notoginseng in the Secondary Prevention for Stroke Patients with "Antiplatelet Drug Resistance"
}

Jie Chen (D 657292134@qq.com )

Shaanxi Provincial Hospital of Traditional Chinese Medicine

Hui Wang

Medical Center

Jie Yuan

Medical Center

Bing Xiao

Medical Center

Bo Lu

Medical Center

Cui Wang

Medical Center

Jin Wang

Medical Center

Cheng Zu Han

Medical Center

Ling Dong Liu

Medical Center

Fan Luo

Medical Center

Yuan Wang

Medical Center

Ying Wang

Medical Center

Ling Lin

Medical Center

Jia Gao

Medical Center

Ting Hu

Medical Center

Chenlin Chao 


\section{Research Article}

Keywords: aspirin resistance, Panax notoginseng, cerebral stroke, platelet aggregation rate, randomized controlled trial, study protocol

Posted Date: October 18th, 2021

DOI: https://doi.org/10.21203/rs.3.rs-649760/v1

License: (c) (1) This work is licensed under a Creative Commons Attribution 4.0 International License. Read Full License 


\section{Abstract}

\section{Background}

Cerebral stroke is common and associated with high rates of mortality, disability, and recurrence while the chance of complete recovery is low. It adversely affects human physical and mental health and represents the leading cause of death in China. Aspirin is the cornerstone of secondary prevention of cerebral stroke. However, recurrence of stroke cannot be completely prevented by regular oral administration of aspirin due to aspirin resistance, which is an independent risk factor for stroke recurrence. In this instance, several strategies have been taken, including dose incrementation, frequency increaseof drug administration, combination with other antiplatelet drugs, or replacing it with new antiplatelet drugs. However, these measures have led to several other undesirable outcomes, including gastrointestinal tract stimulation, increased risk of bleeding, higher cost, and poor patient compliance to treatment.let aggregation, but its efficacy and mechanism in the secondary prevention Numerous studies have confirmed that Panax notoginseng has the effect of anti-plateof cerebral stroke among patients with aspirin resistance have not been ascertained.

\section{Method/Design:}

This is a two-center, triple-blinded, randomized, controlled, and optimal efficiency trial. A total of 120 patients aged 45-65 years old with semi-resistance to antiplatelet drugs treated in the secondary prevention clinics for cerebral stroke in the Shaanxi Traditional Chinese Medicine Hospital and Xi'an Hospital of Traditional Chinese Medicine from October 2020 to June 2022 will be recruited. Patients are randomly divided into the experimental group and control group at the ratio of 1:1, with 60 cases in each group. The blood pressure, blood glucose, and blood lipid are controlled within the normal range as the basic standards of treatment. The experimental group is given aspirin enteric coated tablets $100 \mathrm{mg} / \mathrm{day}$ + Panax Notoginseng Powder ( $\mathrm{g} /$ day, oral administrationafter dissolved), while the control group is given aspirin enteric coated tablets $100 \mathrm{mg} /$ day + placebo (Panax Notoginseng Powder $0.03 \mathrm{~g}+$ malt powder/day, oral administration after dissolved). Measurements on the platelet aggregation rate, thromboxane A2 (TXA $)$, prostacyclin (PGI2), adenosine diphosphate (ADP), and changes of the coagulation index of the two groups are performed on the day-14 and day-30. The efficacy, mechanism, and safety of Panax notoginseng in the treatment of patients with aspirin resistance will be evaluated. The data are analyzed and the mean and confidence interval $(\mathrm{Cl})$ of $95 \%$ level are calculated by the SPSS 21.0 software. The intention-to-treat (ITT) analysis is used to account for the missing data or dropouts. Descriptive analyses are performed on the baseline population data. Repeated ANOVA (general linear model) is used to compare the differences ofkey indicators (platelet aggregation rate, thromboxane $A 2$, prostacyclin, ADP) between the two groups.For the secondary indicators (coagulation function), two independent samples t-test and Wilcoxon rank-sum test are used. $P<0.05$ is considered a statistically significant difference between the two groups. 


\section{Conclusion}

This study aims is to explore the efficacy and mechanism of Panax notoginseng in the secondary prevention for stroke patients with aspirin resistance. The results will provide evidence-based practice for traditional Chinese medicine, and also shed light on how it may influence the secondary prevention of cerebral stroke.

\section{Trial registration:}

The trial has been registered with the Chinese Clinical Trial Registry (http://www.chictr.org.cn/index.aspx,) on 24th October 2020, with the registration number ChiCTR2000037833.

\section{Background}

Cerebral stroke is common with a high incidence rate worldwide. It is associated with a high risk of mortality, disability, and recurrence. The chance of a complete recovery following stroke is unfortunately low. It adversely affects the physical and mental health of patients and has become the leading cause of death in China.Studies have shown that the recurrence rate of stroke among the survivors within 6 months is $8.8 \%$, and the annual recurrence rate is $3-4 \%$, and the symptoms will worsen after each recurrence ${ }^{[1]}$. Thus, antiplatelet drugs form the basis of secondary prevention of stroke to reduce the recurrence rate ${ }^{[2]}$. However, despite regular antiplatelet drugs, stroke recurrence cannot be completely prevented $^{[3-6]}$. In addition to the poor control of other risk factors, resistance to antiplatelet drugs plays an important role in these patients.

Antiplatelet drug resistance is mainly defined by several laboratory indicators that reflect platelet function, as well as the presence of clinical recurrent thrombotic events ${ }^{[5][6]-7]}$. Clinical antiplatelet drug resistance indicates a failure of antiplatelet drugs in reducing the occurrence of embolism and other ischemic events in the secondary prevention of cardiovascular and cerebrovascular diseases, while laboratory antiplatelet drug resistance indicates a failure of inhibition to platelet aggregation function in vitro. Aspirin resistance $(A R)$ and clopidogrel resistance (CR) are common ${ }^{[7]}$. Due to the occurrence of $A R$, the risk of recurrent cerebrovascular events has doubled with the risk of death increased by $3.5 \mathrm{folds}^{[8]}$, which contributes to subsequent heavy economic burden to society and family.

Given that the exact mechanism of AR remains to be studied, currently, there is no specific effective management to AR but mainly focuses on improving patient compliance, controlling other risk factors, increasing aspirin dose, switching to or combining antiplatelet drugs from other pathways, increasing the frequency of drug use, and avoiding drug-drug interactions. However, studies have shown that increasing aspirin dose did not improve the clinical outcomes, but increased the risk of bleeding ${ }^{[9]}$. The use of clopidogrel also shares the problem of resistance to platelet aggregation ${ }^{[10]}$. Although the dual antiplatelet regimen and new antiplatelet drugs show promising efficacy, they have the disadvantages of being high 
cost, increased bleeding risk, irritation to the large gastrointestinal tract, and adverse interaction with other commonly used drugs. Therefore, long-term application of these treatments is limited and an alternative and effective treatment for patients with AR is urgently desired.

Several studies have demonstrated that some traditional Chinese medicine can promote blood circulation and remove blood stasis that may have a good prospect in the treatment of patients with $A R^{[11]-[12]}$. In particular, Panax notoginseng has been shown to disperse stasis and promote blood circulation but also stop bleeding. Through our long-term clinical observation, we have observed the function of Panax notoginseng in easing the platelet aggregation in the secondary prevention of stroke $\mathrm{e}^{[13][14]-15]}$. However, the mechanism and effectiveness of Panax notoginseng in patients with antiplatelet drug resistance remains unknown.

Given the above, this study involves patients with antiplatelet drug resistance in the secondary prevention of stroke as subjects. This would enable the investigation of the mechanism of traditional Chinese medicine as a clinical treatment, allow the determination of the clinical efficacy of Panax notoginseng in the treatment of antiplatelet drug resistance, and provide a solid evidence-based practice for the treatment of antiplatelet resistance in the secondary prevention of stroke in the future.

\section{Method/design}

\subsection{Experimental design}

This is a double-center, triple-blinded, randomized, and optimal efficiency clinical trial. A total of 120 patients with aspirin semi-resistance will be randomized into the experimental group or the control group at the ratio of 1:1, with 60 patients in each group. The experimental group is given enteric-coated aspirin tablets $100 \mathrm{mg} /$ day + Panax Notoginseng Powder (3 g/day, oral administrationafter dissolved), while the control group is given enteric-coated aspirin tablets $100 \mathrm{mg} /$ day + placebo (Panax Notoginseng Powder $0.03 \mathrm{~g}+$ malt powder / day, oral administration after dissolved). The whole trial period is 37 days, inclusive of 7 days of baseline observation and 30 days of treatment. Platelet aggregation rate, $\mathrm{TXA}_{2}$, PGI2, and ADP are measured before treatment, at day-14, and day-30 of the treatment.

\subsection{Ethics}

This study has been approved by the ethics committee of the Shaanxi Traditional Chinese Medicine Hospital ([2019] ethical review No. 08) (http://www.chictr.org.cn/index.aspx) with the registration number of ChiCTR2000037833. Informed consent will be obtained from all subjects. Any alteration of the study protocol or informed consent that may affect the rights and interests of participants or affect the implementation of the study shall be reported to the ethics committee for approval. When any serious adverse events are encountered during the trial, the ethics committee shall timely review and propose a written modification, including full authority to suspend the trial.

\subsection{Research subjects}


2.3.1. Diagnostic criteria for semi-resistance to aspirin. Aspirin resistance is diagnosed as having both the AA-induced platelet aggregation rate $\geq 20 \%$ and ADP-induced platelet aggregation rate $\geq 70 \%$, while those who meet one of the criteria are aspirin semi-resistance ${ }^{[16]}$.

\subsubsection{Inclusion criteria.}

1. $45<$ age $<65$ years old, any gender, taking the single antiplatelet drug (aspirin);

2. More than 2 weeks after the onset;

3. Diagnosed as aspirin semi-resistance;

4.The Essen stroke risk score is less than 6;

5. Platelet count is $100-400 \times 10^{9} / \mathrm{L}$;

6. No tendency of family or individual to bleeding;

7.No history of the myelodysplastic syndrome; no major surgical operation in the past month;

8.Traditional Chinese medicine with the function of promoting blood circulation and removing blood stasis is no longer used in the observation period;

9. Agree to participate in the clinical trial and complete the written informed consent.

\subsubsection{Exclusion criteria.}

1. Patients with a transient ischemic attack or cerebral infarction and have double-resistance;

2. Patients with cerebral hemorrhage after cerebral infarction, cerebral arteritis, brain tumor, brain trauma, and brain parasitic disease; patients with rheumatic heart disease, previous myocardial infarction, obvious cardiac insufficiency, abnormal liver and kidney function, active peptic ulcer, and severe gastrointestinal reaction;

3. Non-menopausal women;

4. Patients with various blood disorders, hemorrhagic diseases, or bleeding tendency, platelet count $>450 \times 10^{9} / \mathrm{L}$ or $<100 \times 10^{9} / \mathrm{L}$, or hemoglobin $<90 \mathrm{~g} / \mathrm{L}$;

5. Patients with an allergic constitution or taking other anti-inflammatory and analgesic drugs (NSAIDs);

6. Patients with disabilities (blindness, deafness, mute, mental retardation, mental disorder, and physical disability caused by other reasons) defined by law.

7. Patients who are suspected or do have a history of alcohol and drug abuse, or who, according to the judgment of the researchers, have other conditions that may reduce the possibility of enrollment or 
complicate their enrollment;

8. Those who are actively participating in other clinical trials or have participated in other clinical trials involving drugs within 3 months.

\subsubsection{Termination criteria.}

1. Violation of inclusion criteria and clinical trial protocol or compliance withexclusion criteria.

2. No treatment for 30 consecutive days, poor compliance, or loss of follow-up.

3. Serious side effects or adverse reactions.

4. Replacement of treatment that may affect the results of the study without the permission of investigators.

5.Withdrawal of consent by the party concerned or the legal representative.

\subsection{Location of study implementation and patient recruitment}

This study will be implemented in the Shaanxi Traditional Chinese Medicine Hospital and Xi'an Hospital of Traditional Chinese Medicine. Patients will be recruited via outpatient, inpatient, official hospital WeChat account, official hospital website, posters, and leaflets.

\subsection{Experimental grouping}

All patients successfully recruited will be randomized into either the experimental group or the control group according to the ratio of 1:1, with 60 cases in each group.

\subsection{Test time}

From $1^{\text {st }}$ October 2020 to $30^{\text {th }}$ September 2022.

\subsection{Interventions}

All the researchers were clinical doctors who have obtained the qualification certificate of medical practitioners issued by the National Health and Family Planning Commission, and have more than three years of clinical experience.

2.7.1. Treatment in the Control group.Basic treatment + placebo (a total of $3 \mathrm{~g}$, comprised of $0.3 \mathrm{~g}$ Panax Notoginseng Powder and 2.7g malt powder, once a day, taken orallyafter dissolved)

2.7.2. Treatment in the experimental group. Basic treatment + Panax Notoginseng Powder (3g, once a day, taken orallyafter dissolved) 
Basic treatment: The secondary prevention plan forcerebral stroke is implemented according to the Guidelines for Prevention and Treatment of Cerebrovascular Diseases in China (2014 Edition).Briefly:

(1)Antiplatelet aggregation: Oral administration of enteric-coated aspirin tablets $100 \mathrm{mg}$, once a day.

(2) Hypertension: Calcium channel antagonists (e.g., nifedipine), ACEl (e.g., Enalapril), ARB (e.g., Valsartan), $\beta$-blockers (e.g., metoprolol), and diuretics (e.g., hydrochlorothiazide) are selected according to the patient's condition to optimize blood pressure with the target of $\leq 140 / 90 \mathrm{mmHg}$, and it can be reduced to $\leq 130 / 80 \mathrm{mmHg}$ under the condition of tolerance.

(3) Abnormal lipid metabolism: Long-term treatment with simvastatin, atorvastatin, or rosuvastatin with the target value of LDL-C decrease $\geq 50 \%$ or $L D L \leq 1.8 \mathrm{mmol} / \mathrm{L}$.

(4)Diabetes mellitus: Insulin or oral hypoglycemic drugs are given, and the overall goal is $\mathrm{HbA} 1 \mathrm{C} \leq 7 \%$.

(5)Hyperhomocysteinemia: Oral administration of folic acid tablets $0.4 \mathrm{mg}$, once a day; mecobalamin capsules $0.5 \mathrm{mg}, 3$ times a day.

\subsection{Observation of adverse events}

Some patients may experience dry lips, restlessness, bleeding tendency in different parts of the body.

\subsection{Primary indicators}

Blood samples will be collected from the elbow vein before treatment, at day-14, and day-30 of treatment. The platelet aggregation function, TXB2, PGI2, and ADP will be monitored.

\subsection{Secondary indicators}

The changes in blood pressure, blood lipid, and blood glucose are recorded before treatment, at day-14, and day-30 of treatment, when the changes of coagulation function are also monitored. The liver and kidney functions are monitored before and after the treatment to ensure safety.

\subsection{Sample size estimation}

This is a preliminary study that aimed to evaluate the efficacy and mechanism of the treatment. A total of 120 participants will be recruited.

\subsection{Randomization}

The random distribution method will be operated by the coordinator of the Department of Encephalopathy of Shaanxi Traditional Chinese Medicine Hospital. Random numbers are generated by the SPSS 21.0 software and grouped by unpredictable random sequence block design. Patients are randomly assigned to the control group or the treatment group according to the $1: 1$ ratio. The serial 
number will be sealed in an opaque envelope and managed by PI. The random sequence block will not be informed to the research object or the implementer.

\subsection{Statistical analysis}

Data analysis will account for the lack of data through the principle of intentionality. Continuous variables that conform to normal distribution or approximate normal distribution are represented by mean \pm standard deviation $( \pm s)$, while continuous variables that do not conform to normal distribution are represented by median and quartile [M (P25, P75)]. Categorical variables are described by frequency or percentage. The Chi-square test is used to compare disordered classified variables between groups, and the rank-sum test is used for ordered classified variables.Continuous variables are analyzed by t-test, corrected t-test, or rank-sum test of two samples; repeated measurement data are analyzed by repeated measurement ANOVA; if the conditions of repeated measurement ANOVA are not met, generalized estimation equation analysis will be used; if there is a statistical difference between groups and there is interaction, the single effect analysis will be conducted. All tests are bilateral, with a P-value of $<0.05$ considered statistically significant.

2.13.1.Results of primary indicators. Univariate repeated measures ANOVA will be used to compare the differences in platelet aggregation rate, $\mathrm{TXA}_{2}, \mathrm{PGI} 2$, and ADP between the two groups before treatment, at day- 14 , and day-30 of treatment.

2.13.2. Results of secondary indicators.Univariate repeated measurement ANOVA will be used to compare the general condition, blood pressure, blood lipid, coagulation function, and blood glucose between the two groups before treatment, at day-14, and day-30 of treatment.

2.13.3.Safety assessment. The T-test will be used to compare the liver and renal functions before treatment and at day-30 after treatment.

\subsection{Quality control}

The withdrawals and reasons for withdrawal will be fully documented throughout the treatment and follow-up period. To ensure the quality of the study, the quality monitoring will periodically verify all process details and check the authenticity of the data. The Scientific Research Department of Shaanxi Traditional Chinese Medicine Hospital has been invited as the third party to manage the data independently.Given that differences between physicians may result in biases, each assessment is performed by a designated physician who has been trained in the same assessment criteria. Before recruitment, doctors in charge of the treatment will receive special training on research and operation, including randomization, blinding, and key points of diagnosis and treatment. To record the participant's attendance and compliance, each participant has been made a record card that records the date of treatment, personal information, and the signature of the patient after each treatment.

\section{Discussion}


The occurrence of aspirin resistance leads to a doubled increased risk of thromboembolic events ${ }^{[17]}$, especially the recurrence of stroke, which has exerted a significant socioeconomic burden to the society and families. The mechanism of aspirin resistance is complex. Studies have shown that patient compliance with treatment, gender, inflammation, hypertension, diabetes and other clinical, genetic, pharmacological, and biological factors can contribute to aspirin resistance ${ }^{[18][19][20][21][22]-23]}$.Due to the complexity and multi-factorial of the pathogenesis, there is no consensus on its treatment. Therefore, this study has been designed to obtain high-quality data through the advantages of multi-target and multichannel of traditional Chinese medicine, which provide a reliable basis for future multi-center research.

This study includes patients with aspirin semi-resistance in the secondary prevention of stroke. Given that the treatment of aspirin resistance remains controversial, the control group is treated with aspirin + placebo, and the observation period of 30 days and Essen stroke risk score $<6$ points have been utilized to reduce the recurrence rate of stroke. On the other hand, the experimental group is treated with aspirin + Panax notoginseng, which can promote blood circulation and also stop bleeding, reduce blood stasis, and dredge collaterals. With its anti-platelet aggregation effect, Panax notoginseng has been widely used in patients with stroke, coronary heart disease, lower extremity vascular occlusion, and other thromboembolic diseases. However, the effects of Panax notoginseng in reducing the platelet aggregation rate and lowering the risk of disease recurrence among patients with aspirin semi-resistance remains unclear.In this study, the adoption of placebo-controlled and triple-blind methodology permits the efficacy of Panax notoginseng to be ascertained in the treatment of stroke patients with aspirin semiresistance, while the possible mechanisms of its effects can also be explored. The results of this study will provide high-quality evidence for the use of Panax notoginseng in clinical practice.

This is a preliminary study, which may be limited by a short observation period, no set of the follow-up period, and the dose-effect relationship between high, medium, and low doses of Panax notoginseng are not investigated.

\section{Declarations}

\section{Ethical approval}

The trial protocol has been approved by the Independent Ethics Committee of Shaanxi Province Hospital of Traditional Chinese Medicine on October 24, $2020((2019)(08))$. This trial was registered at Chinese Clinical Trial Registry(ChiCTR2000037833) on October 24,2020.The informed consent forms must obtained from each patient.

\section{AvailAvailability of data and materialsability of data and materials}

Not applicable

\section{Consent for publication}


Not applicable

\section{Conflict of interest}

All the author declare that they no conflict of interest to declare.

\section{Funding}

Not applicable

\section{Author contributions}

Jie Chen and Hui Wang designed this study together. Hui Wang,Jie Yuan and Jie Chen drafted the protocol.Bing Xiao performed the statistical analysis.Bo Lu was responsible for the writing revision.All authors read and approved the final manuscript.

Data collection:Cui Wang,Ying Wang,Ling Lin,Jia Gao,Ting Hu,Chenlin Yao.

Formal analysis:Hui Wang,Fan Luo,Yuan Wang.

Funding acquisition:Jie Chen,Jie Yuan.

Methodology:Jie Chen,Jin Wang.

Project administration:Jie Chen.

Project coordination:Zucheng Han,Dongling Liu.

Writing-original draft:Hui Wang,Jie Yuan,Jie Chen.

Writing-review \& editing: Bing Xiao,Bo Lu.

Consent for publication: Agree to publish.

\section{Author information}

${ }^{\square}$ Tianjin University of Traditional Chinese Medicine, Tianjin City,

${ }^{b}$ Department of Encephalopathy, Shaanxi Hospital Province of Traditional Chinese Medicine, Xi 'an囚

cXi 'an Hospital of Traditional Chinese Medicine, Xi 'an, Shaanxi Province,

${ }^{\mathrm{d}}$ School of Basic Medical Sciences, Chengdu University of Traditional Chinese Medicine $\mathbb{Z}$

e First Affiliated Hospital of Xi 'an Medical College,

f Shaanxi University of Traditional Chinese Medicine, Xianyang, Shaanxi Province, P.R. China. 
${ }^{g}$ These authors contributed equally.

*Correspondence: Jie Chen, Department of Encephalopathy, Shaanxi Hospital Province of Traditional Chinese Medicine,No.4 Xi-huamen, Xi 'an, 710003, Shaanxi, Province, P.R. China(email:657292134@qq.com)

\section{References}

1. Floyd CN,Ferro,A.Mechanisms of Aspirin Resistance[J].Pharm acology and Therapeutics, 2014,141(1).

2. Chinese Society of Cardiology, Chinese Medical Association. Editorial Board of Chinese Journal of Cardiology. Chinese expert consensus on antiplatelet therapy [J]. Chinese journal of cardiovascular diseases,2013,41.

3. He YR. the Party loves the people. Advances in aspirin and clopidogrel resistance and gene polymorphism [J]. Chin J hypertension. 2017;25(11):1084-6.

4. Song JJ, Bai HY. The role of aspirin resistance and platelet activation in cerebral infarction recurrence [J]. Chinese journal of applied neurological diseases. 2016;19(10):87-8.

5. Yu JH, Wang YL. Antiplatelet drug resistance and recurrent ischemic stroke [J]. Chinese journal of applied neurological diseases. 2017;20(16):113-6.

6. Li XG, Ling X, Zhao K, et al. Research progress of anti-platelet drug resistance [J]. Drug evaluation analysis in Chinese hospitals. 2017;17(3):289-96.

7. Jing LC, Sun RH. Studies on the resistance mechanism of aspirin [J]. Journal of brain and neurological diseases,2017,25(10),657-660.

8. Urbanowicz T, Komosa A,Michalak M,et al.The incidence of aspirin resistance in heart transplantation recipients[J]Kardiochir Torako chrurgia Pol,2017;14(2):115-9.

9. Wang M, Xue M, Xu Y. Panax notoginseng saponin is superior to aspirin in inhibiting platelet adhesion to injured endothelial cells through COX pathway in vitro[J].Thrombosis Research,2016,141.

10. El-Mitwalli A, Azzam H, Abu-Hegazy M,Gomaa M,Wasel Y.Antiplatelets in secondary stroke prevention.Antiplatelet Therapy:Aspirin Resistance and All That. Jazz![J].Thromb Haemost,2016,10(12):1126-1134.

11. Zhang ZY, Huang Y, Ma J, et al. The correlation research between blood stasis syndrome and antiplatelet drug resistance in patients with ischemic stroke [J]. Journal of integrated traditional Chinese western medicine of cardio-cerebrovascular disease. 2018;16(4):472-4.

12. Mao W,Current situation and prospect of integrated traditional Chinese and western medicine for prevention and treatment of thrombosis events [J]. Journal of integrated traditional Chinese and western medicine for cardio-cerebrovascular diseases,2016,14 (5): 449-453. 
13. Yang JJ, Liu Y, Wang CX, et al. Modern research progress of notoginseng saponin r-1 [J]. Chinese journal of hospital pharmacy. 2015;35(5):463-7.

14. Wang Y, Zhu Y, LI W, et al. Research progress on saponins in panax notoginseng and pharmacological effects [J]. Chinese herbal medicine. 2015;46(9):1381-92.

15. Zhong XF. Pharmacological action of panax notoginseng and its clinical application [J]. Clinical research of traditional Chinese medicine. 2015;7(6):116-7.

16. CaoJ,Liu. L,Fan,L,eta1.1eprevalence risk factors and prognosis of aspirin resistance inelderly male patients with cardiovascular disease[J]. Aging Male. 2012;15(3):140-7.

17. Zhang N. Wang Z,Zhou L.Aspirin resistance are associated with long-term recurrent stroke events after ischaemic stroke[J]. Brain research bulletin. 2017;134:205-10.

18. Floyd CN. Ferro A.mechanism of asprin resistance[J]. Pharmacology Therapetutics. 2014;141(1):6978.

19. E.Yalcinkaya. M.Celik,Evaluation of inflammatory conditions associated with aspirin resistance,Ups. JMedSci. 2014;119(2):292-3.

20. Yalcinkaya E,Murat Celik.Evaluation of inflammatory conditions associated with aspirin resistance [J],Ups.J.Med.Sci.2014.119.29 2-293.

21. Tayfur, Toptas,Eyyup Tasdemir,Author's reply: Predictors of aspirin resistance[J],Ups.J.Med.Sci.2014.119.294.

22. Naro C, Sette C. Dissecting a Hub for Immune Response:Modeling the Structure of MyD88[J].Structure,2016,24(3):349-51.

23. Gong $X$, Wang $X ; X u, Z$.Over-expression of cyclooxygenase-2 in increased reticulated platelets leads to aspirin resistance after elective off-pump coronary artery bypass surgery[J].Thrombosis Research,2017,160.

\section{Supplementary Files}

This is a list of supplementary files associated with this preprint. Click to download.

- CONSORTchecklist.docx 